\title{
Extending the concept of edge collection function to polygonal and curved planar luminescent waveguides
}

\author{
Ichiro Fujieda* and Yasuhiro Tsutsumi \\ Ritsumenkan University, Department of Electrical and Electronic Engineering, Kusatsu, \\ Shiga, Japan
}

\begin{abstract}
When a single spot in a planar luminescent waveguide is excited, some photoluminescence photons that are trapped inside reach its edge. Considering the photon losses due to leakage from its top and bottom surfaces and self-absorption during wave-guiding, the probability of collecting photons at its edge is expressed as a function of the coordinates of the excited spot for polygonal and curved waveguides. The emission is assumed to be isotropic, and scattering and re-emission events are neglected for simplicity. This model might be useful for predicting the performance of luminescent waveguides with various shapes under non-uniform illumination. (c) The Authors. Published by SPIE under a Creative Commons Attribution 4.0 Unported License. Distribution or reproduction of this work in whole or in part requires full attribution of the original publication, including its DOI. [DOI: 10.1117/1.JPE.10.044501]
\end{abstract}

Keywords: waveguide; photoluminescence; optical efficiency; edge collection function; luminescent solar concentrator.

Paper 20046 received Jun. 2, 2020; accepted for publication Sep. 28, 2020; published online Oct. $12,2020$.

\section{Introduction}

A luminescent solar concentrator (LSC) harvests energy from sunlight with the solar cells attached to the edges of a waveguide in which luminescent materials are embedded. ${ }^{1,2}$ The materials are either dispersed uniformly in a waveguide or coated on its surface. They can also be contained in a thin layer sandwiched by a pair of transparent plates. We refer to such a configuration as a luminescent waveguide (LWG) in this paper. The materials inside an LWG convert incoming radiation to photoluminescence (PL) photons, some of which reach the solar cells. Due to the overlap between the emission and absorption spectra of these materials, some PL photons are absorbed by the luminescent materials. This self-absorption problem has been recognized since the early days of LSC research. ${ }^{3}$ Although many studies aim to develop overlapfree materials, ${ }^{4-6}$ the highest power conversion efficiency of an LSC remains at 7.2\% measured with a small test device. ${ }^{7}$ Aside from large-scale power generation, semi-transparent LWGs with various colors have an aesthetic advantage in building-integrated photovoltaics (BIPV). ${ }^{8}$ Furthermore, potential applications of LWGs include energy-harvesting displays, ${ }^{9-11}$ sensors for visible light communication, ${ }^{12}$ gamma-ray astronomy, ${ }^{13}$ and position-sensitive detectors. ${ }^{14}$

Optical efficiency of an LWG is important for these applications. It is defined as the probability with which the PL photons reach the LWG edge. Numerical integration ${ }^{1,2}$ and Monte Carlo simulation ${ }^{3,15}$ have been used to estimate the optical efficiency of LSCs for many years. Recently, analytical expressions with a simplifying assumption on the spectral dependency of self-absorption events were derived. ${ }^{16}$ Note that the radiation pattern incident on an LSC is assumed to be uniform. This assumption does not hold in some cases. For example, when it is installed on a building wall in an urban area, surrounding structures can block the sunlight. The intensity pattern of diffused light fluctuates if the surrounding objects and light sources are not stationary. For display and sensor applications of LWGs, the incident light pattern is not uniform in nature. For handling arbitrary incident patterns, the response of an LWG to a single-spot excitation is required. We expressed the optical efficiency of a planar LWG as a function of the coordinates of the excited spot and referred it as edge collection function (ECF). ${ }^{17}$ Due to the crude geometric assumptions in this model, its applicability is limited to a square LWG.

*Address all correspondence to Ichiro Fujieda, fujieda@se.ritsumei.ac.jp 
An early study on an LSC reported that optical efficiency is relatively independent of the shape. ${ }^{18}$ Although the LWGs studied for LSCs are mostly rectangular, other shapes such as a triangle, a hexagon, ${ }^{19-21}$ a fiber, ${ }^{22,23}$ a cylinder, ${ }^{24,25}$ and a leaf ${ }^{26}$ have been reported. A small heart-shaped LWG might have an aesthetic value to be incorporated in a wearable electronic device.

In this paper, we analyze PL photon propagation in a planar LWG after single-spot excitation. In Sec. 2, optical efficiency is formulated in cylindrical geometry. The resultant efficiency as a function of the cylinder radius is converted to one in the Cartesian coordinate system, and ECFs are calculated for polygonal and curved LWGs in Sec. 3.

\section{Optical Efficiency in Cylindrical Geometry}

When a single spot on a thin luminescent layer in a planar waveguide is excited, a concentric emission pattern appears. This is reproduced by a model based on self-absorption and subsequent re-emission of PL photons inside the waveguide. ${ }^{27}$ We extend this model to formulate optical efficiency of a planar LWG in cylindrical geometry. Some numerical examples are given.

\subsection{Model}

In this analysis, we assume that the photon emission from its luminescent material is isotropic. Self-absorption and leakage during the wave-guiding process are accounted for. Scattering is neglected for simplicity. Furthermore, we neglect re-emission events based on our previous experiment. ${ }^{27} \mathrm{~A}$ detailed argument behind this assumption is provided in the Appendix.

Let us consider a planar LWG of radius $R$ and thickness $\ell$ as shown in Fig. 1. Suppose that the center of the luminescent layer is excited by a monochromatic light at wavelength $\lambda_{\mathrm{ex}}$. Let $N_{\text {total }}$ be the total number of PL photons exiting the luminescent layer. Some PL photons escape the waveguide from its top and bottom surfaces without being reflected at the air-waveguide interface. Let this number be $N_{\text {escd }}$. Among those reflected back, some are absorbed by the luminescent layer and some escape from the top and bottom surfaces during the wave-guiding process. Let these numbers be $N_{\mathrm{abs}}$ and $N_{\text {esc }}$, respectively. Those surviving these loss mechanisms reach the edge. Hence, the optical efficiency is expressed as follows:

$$
\eta=\frac{N_{\text {total }}-N_{\text {escd }}-N_{\text {esc }}-N_{\text {abs }}}{N_{\text {total }}}
$$

In the case of modeling an LSC, reflectance of the PL photons emitted within the so-called escape-cone is often assumed to be zero. Outside the escape-cone, the reflectance is unity due to total internal reflection (TIR). It is worth noting that this stepwise treatment is not applicable for calculating an ECF. This is readily understood by considering the photon emitted from the region very near the edge of an LWG. It has a higher probability of reaching its edge because its escape-cone extends sideway as well. The stepwise approach would significantly underestimate the optical efficiency of an LWG when its peripheral region was excited. Therefore, we need to consider the photons escaping the LWG with and without reflection separately.

Each photon count in the right side of Eq. (1) is formulated below. We assume that the emission of PL photons from a luminescent material is isotropic. However, because the material is

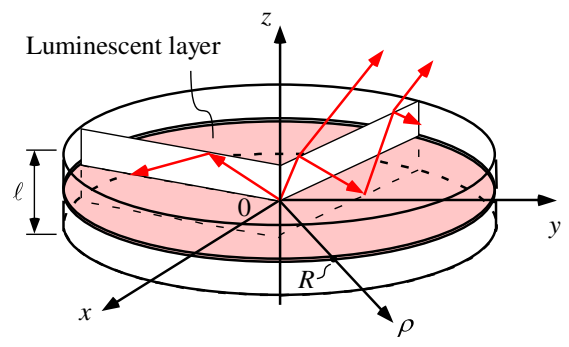

Fig. 1 Cylindrical waveguide with an embedded luminescent layer. 


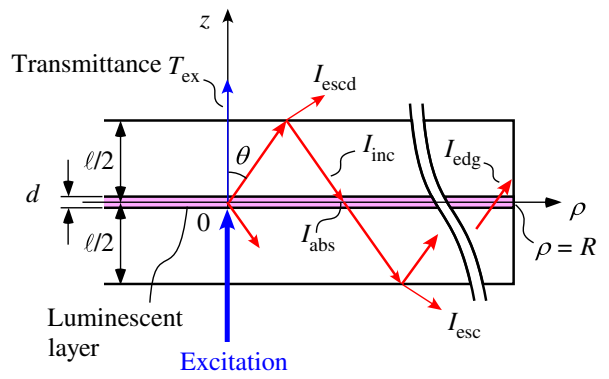

Fig. 2 Cross section of an LWG and spectral intensities of interest.

self-absorbing, the fluxes exiting forward and backward depend on the emission angle $\theta$ as defined in Fig. 2. Denoting the linear absorption coefficient of the layer at wavelength $\lambda$ as $\mu(\lambda)$, the angular intensity distributions of the forward and backward fluxes are expressed as follows: $:^{28}$

$$
\begin{aligned}
I_{f}=\frac{1}{2 \pi} \eta_{\mathrm{QE}} S_{\mathrm{em}}(\lambda)\left(1-T_{\mathrm{ex}}\right) S_{0} \frac{T_{\mathrm{ex}}^{\alpha}-T_{\mathrm{ex}}}{1-\alpha}, & 0 \leq \theta \leq \frac{\pi}{2}, \\
I_{b}=\frac{1}{2 \pi} \eta_{\mathrm{QE}} S_{\mathrm{em}}(\lambda)\left(1-T_{\mathrm{ex}}\right) S_{0} \frac{1-T_{\mathrm{ex}}^{1+\alpha}}{1+\alpha}, & \frac{\pi}{2} \leq \theta \leq \pi .
\end{aligned}
$$

Here, we introduced the parameters quantum efficiency of the luminescent material $\eta_{\mathrm{QE}}$, its emission spectrum $S_{\mathrm{em}}(\lambda)$, and the strength of the excitation light $S_{0}$. The factor $\frac{1}{2 \pi}$ has been added to the expressions in Ref. 28 to account for the light spreading in the azimuthal angle in the current three-dimensional analysis.

Denoting the thickness of the luminescent layer as $d$, the parameters $\alpha$ and $T_{\text {ex }}$ are defined as follows:

$$
\begin{gathered}
T_{\mathrm{ex}}=e^{-\mu\left(\lambda_{\mathrm{ex}}\right) d}, \\
\alpha=\frac{\mu(\lambda)}{\mu\left(\lambda_{\mathrm{ex}}\right)} \cdot \frac{1}{\cos \theta} .
\end{gathered}
$$

First, let us express the total spectral flux exiting the luminescent layer $F_{\text {total }}$ by considering a unit sphere around the excited spot. The luminescent layer is assumed to be thinner than the diameter of this sphere. Integrating the spectral intensity $I_{f}+I_{b}$ by the azimuthal angle and folding the range of the polar angle in half, $F_{\text {total }}$ is expressed as

$$
F_{\text {total }}=2 \pi \int_{0}^{\pi / 2}\left(I_{f}+I_{b}\right) \sin \theta \mathrm{d} \theta .
$$

Second, for expressing the photon count $N_{\text {escd }}$, the spectral intensity $I_{f}+I_{b}$ is multiplied by the transmittance $\left(1-R_{F}\right)$, where $R_{F}$ is the reflectance given by the Fresnel equations and the upper limit for the integration by the polar angle is set to $\theta_{b} \equiv \tan ^{-1} \frac{R}{\ell / 2}$ :

$$
F_{\text {escd }}=2 \pi \int_{0}^{\theta_{b}}\left(1-R_{F}\right)\left(I_{f}+I_{b}\right) \sin \theta \mathrm{d} \theta
$$

Third, we analyze the photon losses during the wave-guiding process. As for the photon count $N_{\text {abs }}$, our previous analysis ${ }^{27}$ is adopted as follows. Let us express the PL photon intensity incident on the luminescent layer at $\rho=i \ell \tan \theta_{i}$, where $i$ is the number of reflections. The forward and backward fluxes need to be transmitted through the luminescent layer $i-1$ times. They need to be reflected by the waveguide surfaces $i$ times. The transmittance $P_{t}$ and absorbance $P_{a}$ for the PL photon propagating with angle $\theta_{i}$ are given as 
Fujieda and Tsutsumi: Extending the concept of edge collection function to polygonal...

$$
\begin{gathered}
P_{t}=e^{-\mu(\lambda) \frac{d}{\cos \theta_{i}},} \\
P_{a}=1-P_{t} .
\end{gathered}
$$

In addition, the spectral intensity $I_{f}+I_{b}$ is reduced by the factor $1 /\left\{\rho^{2}+(i \ell)^{2}\right\}$ because of the spreading over the distance $\sqrt{\rho^{2}+(i \ell)^{2}}$. Hence, the spectral intensity incident at $\rho=i \ell \tan \theta_{i}$ is given by $P_{t}^{i-1} R_{F}^{i} \times \frac{I_{f}+I_{b}}{\rho_{i}^{2}+(i \ell)^{2}}$. Because this is projected on the luminescent layer with angle $\theta_{i}$, the projection factor $\cos \theta_{i}$ is multiplied. Adding contributions from each $\theta_{i}$, the following expression holds for the total intensity of the spectral flux absorbed at $\rho_{i}=i \ell \tan \theta_{i}$.

$$
I_{\mathrm{abs}}=\sum_{i=1}^{\infty} P_{a} P_{t}^{i-1} R_{F}^{i} \times \frac{I_{f}+I_{b}}{\rho_{i}^{2}+(i \ell)^{2}} \times \cos \theta_{i} .
$$

As for the photon count $N_{\text {esc }}$, this is slightly modified to give the following expression for the total spectral intensity of the PL photons escaping the waveguide at $\rho_{i}=i \ell \tan \theta_{i}$ :

$$
I_{\mathrm{esc}}=\sum_{i=1}^{\infty} P_{t}^{i-1} R_{F}^{i-1} \times \frac{I_{f}+I_{b}}{\rho_{i}^{2}+(i \ell)^{2}} \times \cos \theta_{i} \times\left(1-R_{F}\right) .
$$

The total spectral fluxes lost during the wave-guiding process in an LWG of radius $R$ are calculated by integrating these intensities as follows:

$$
\begin{aligned}
& F_{\mathrm{abs}}=2 \pi \int_{0}^{R} I_{\mathrm{abs}} \rho \mathrm{d} \rho, \\
& F_{\mathrm{esc}}=2 \pi \int_{0}^{R} I_{\mathrm{esc}} \rho \mathrm{d} \rho .
\end{aligned}
$$

Once these spectral fluxes are known, the corresponding photon counts are given by integrating with respect to the wavelength. In the numerical example below, we resort to numerical integration (Simpson's method) for Eqs. (6) and (7). The maximum number for the summation in Eqs. (10) and (11) is increased to 50 although 10 is justified for convergence for the same input parameter set. $^{27}$

\subsection{Numerical Example}

Lumogen F Red 305, a standard luminescent material applied for an LSC, is assumed for the examples in this paper. The normalized absorption and emission spectra in Fig. 3 are reproduced from the data in Ref. 29. Other input parameters are assumed as follows: the quantum efficiency of this material $\eta_{\mathrm{QE}}=1,{ }^{30}$ the index of refraction $n=1.5$, and $\lambda_{\mathrm{ex}}=450 \mathrm{~nm}$.

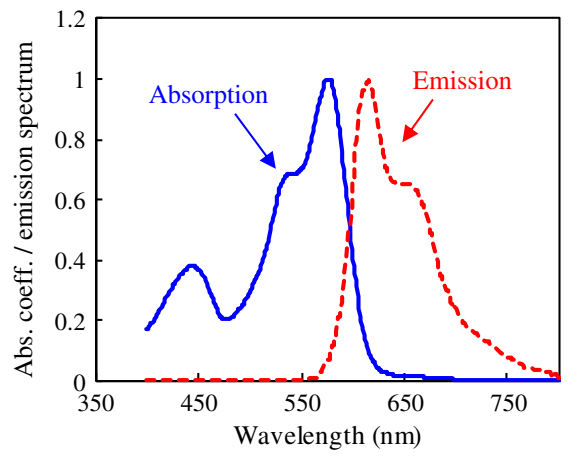

Fig. 3 Normalized absorption coefficient and emission spectrum of Lumogen $F$ Red 305 $(\mathrm{BASF}){ }^{29}$ 
Fujieda and Tsutsumi: Extending the concept of edge collection function to polygonal...
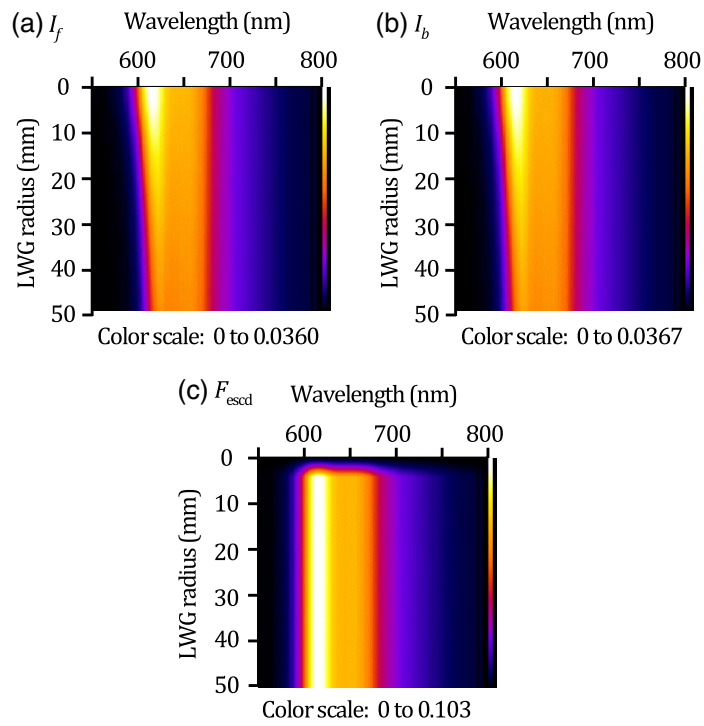

Fig. 4 The spectral intensities $I_{f}, I_{b}$, and the flux $F_{\text {escd }}$ calculated for 10-mm-thick LWGs with $T_{\text {ex }}=0.1$. (a) The forward intensity is smaller than (b) the backward intensity and more severely redshifted. (c) The flux escaping without reflections is constant for the LWGs with $R \geq \frac{\ell}{2} \tan \theta_{c}$.

First, the spectral intensities $I_{f}, I_{b}$, and the flux $F_{\text {escd }}$ are calculated as a function of the LWG radius $R$ by Eqs. (2), (3), and (7), respectively. The result is shown in each color-coded image in Fig. 4. In this example, the thickness $\ell$ and the transmittance $T_{\text {ex }}$ are fixed at $10 \mathrm{~mm}$ and 0.1 , respectively. In each image, a color scale is shown at the far right end and its range is indicated below. Because of the factor $S_{0}$ in Eqs. (2) and (3), the unit for the values in these images is arbitrary. When we calculate optical efficiency, this factor cancels out. The color scale is adjusted to show subtle changes in the data in each image. For example, the maximum value of $I_{f}$ in Fig. 4(a) is 0.0360 and that of $I_{b}$ in Fig. 4(b) is 0.0367 . This convention will be used in all of the color-coded figures in this paper.

It is worth noting that $I_{f}$ is smaller than $I_{b}$ and is more severely redshifted due to selfabsorption. Because of TIR, $F_{\text {escd }}$ remains constant for the LWGs with $R \geq \frac{\ell}{2} \tan \theta_{c}$, where $\theta_{c}$ is the critical angle for TIR. The LWGs with smaller radii exhibit lower $F_{\text {escd }}$ because the escape-cone is not fully developed. Consequently, the ECF values in the peripheral region of an LWG are enhanced, as will become clear in Sec. 3.

Second, the spectral intensities $I_{\mathrm{esc}}$ and $I_{\mathrm{abs}}$ are calculated for two LWGs. The result is shown in Fig. 5 in the same manner. The thickness $\ell$ is set to either 10 or $4 \mathrm{~mm}$. The transmittance $T_{\text {ex }}$ is fixed at 0.1 .

The distributions in Fig. 5 peak at the integer multiples of $\ell \tan \theta_{c}$ because the reflectance $R_{F}$ changes abruptly around $\theta_{c}$. This behavior of $I_{\text {abs }}$ is the origin of the concentric re-emission pattern. ${ }^{27}$ It is worth noting that $I_{\text {esc }}$ and $I_{\text {abs }}$ are complimentary in a sense that more leakage from the waveguide at a certain radius means less absorption there. This comes from the factors $R_{F}$ and $1-R_{F}$ in Eqs. (10) and (11), respectively. As the radius increases, $I_{\mathrm{esc}}$ approaches zero, indicating that PL photons with $\theta<\theta_{c}$ are quickly depleted. The redshift in $I_{\mathrm{abs}}$ is more severe for the 4-mm-thick LWG due to the longer distance that the PL photons propagate inside the LWG. The spectral intensity $I_{\text {abs }}$ is almost zero at the wavelength larger than about $700 \mathrm{~nm}$, simply indicating that these PL photons reach the edge without self-absorption.

The photon counts in the right side of Eq. (1) are given by integrating these spectral intensities, and optical efficiency is calculated as a function of the LWG radius. This is repeated for LWGs with $\ell=10$ and $4 \mathrm{~mm}$. The transmittance of the excitation light $T_{\mathrm{ex}}$ is varied as a parameter. As shown in Fig. 6, optical efficiency $\eta$ is close to unity for LWGs with $R \ll \ell$ because the edge of the cylinder subtends a much larger solid angle than the top and bottom surfaces. As the radius $R$ increases, $\eta$ decreases steeply because $N_{\text {escd }}$ increases rapidly. Because self-absorption becomes the dominant loss mechanism for $R>\frac{\ell}{2} \tan \theta_{c}, \eta$ gradually decreases. As $T_{\mathrm{ex}}$ 


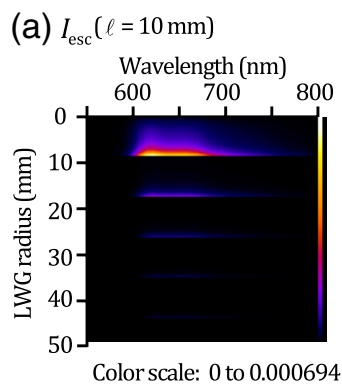

(b) $I_{\text {abs }}(\ell=10 \mathrm{~mm})$
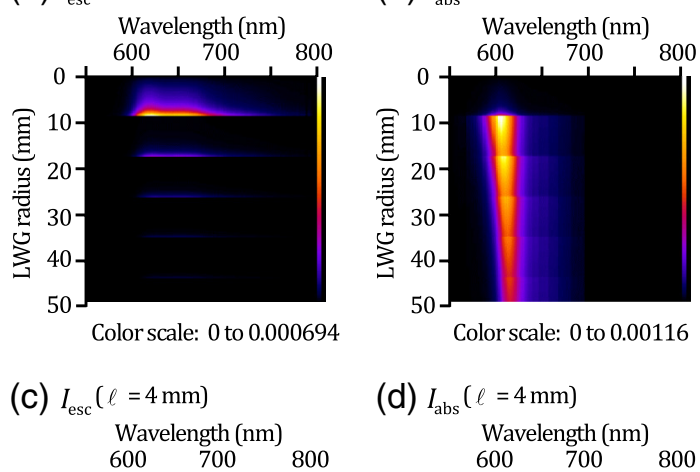

(d) $I_{\text {abs }}(\ell=4 \mathrm{~mm})$

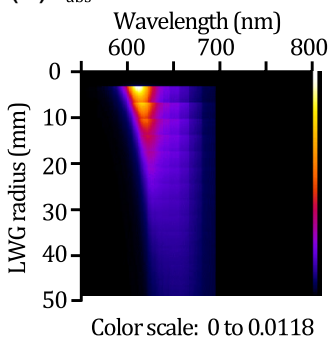

Fig. 5 The spectral intensities after at least one reflection. (a) The intensity $l_{\text {esc }}$ and (b) the intensity $l_{\text {abs }}$ for a $10-\mathrm{mm}$-thick LWG. They have peaks at around 9 and $18 \mathrm{~mm}$. The peaks for a $4-\mathrm{mm}-$ thick LWG in (c) and (d) are shifted toward the excited spot at the origin.

increases, this decrease becomes more gradual. For the extreme case of $T_{\mathrm{ex}}$ near unity, $\eta$ approaches to $\cos \theta_{c}$, which is the trapping probability of the light emitted by an isotropic point source. Nevertheless, a small value of $T_{\mathrm{ex}}$ is not ideal for energy-harvesting because the incident light just passes through the LWG. The effect of $\ell$ on $\eta$ is apparent in Fig. 6, indicating how selfabsorption during the wave-guiding process plays a role in determining $\eta$.

\section{Edge Collection Function}

In this section, we first take an example of a square LSC to show the procedure of calculating an ECF. Then, it is applied for some LSCs with unconventional shapes.

\subsection{Calculation Procedure}

First, the radial distribution $\eta(R)$ is converted to $\eta(x, y)$ in the Cartesian coordinate system. As shown in Fig. 7(a), a mask is set up to specify the shape of an LSC and its photon-collecting edge (in this case, a square and its bottom edge, respectively). The efficiency distribution is translated under this mask. When the source is located at $\left(X_{0}, Y_{0}\right)$ in the coordinate system in Fig. 7(a), the number of photons collected by the bottom edge is given by integrating $S_{o} \times \eta\left(x-X_{0}, y-Y_{0}\right)$ along this edge, where $S_{o}$ is the strength of this source. The total number of photons emitted by the source is given by integrating $S_{o}$ along the whole perimeter of this square. Because $S_{o}$ is canceled, the ECF is expressed as

$$
\operatorname{ECF}\left(X_{0}, Y_{0}\right)=\frac{\int_{C E} \eta\left(x-X_{0}, y-Y_{0}\right) \mathrm{d} s}{\int_{W P} 1 \mathrm{~d} s},
$$

where the photon-collecting edge and the whole perimeter are denoted as $C E$ and $W P$, respectively. By shifting the efficiency distribution on the mask and evaluating the two line integrals in Eq. (14), the ECF for all points within the square mask is calculated. This procedure can be applied for calculating ECFs for various shapes. For example, the frame in Fig. 7(b) and its section in Fig. 7(c) are used in Sec. 3.2 for calculating ECFs of a heart-shaped LWG. 
Fujieda and Tsutsumi: Extending the concept of edge collection function to polygonal...
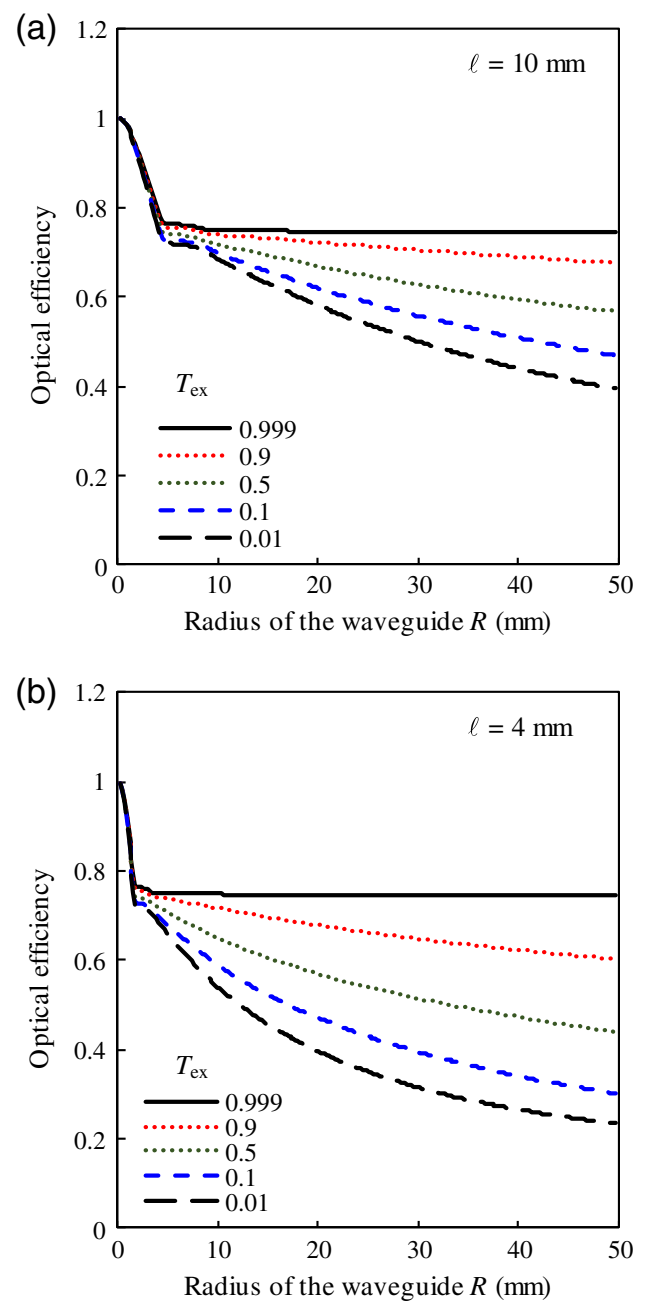

Fig. 6 The optical efficiency calculated for cylindrical LWGs. The thickness $\ell$ is set to (a) 10 and (b) $4 \mathrm{~mm}$. The transmittance of the excitation light $T_{\mathrm{ex}}$ is varied as a parameter.

(a)

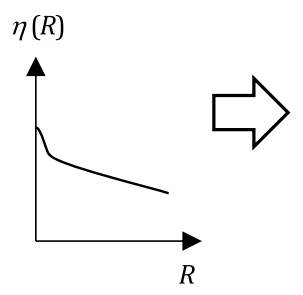

(b)

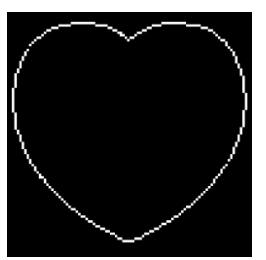

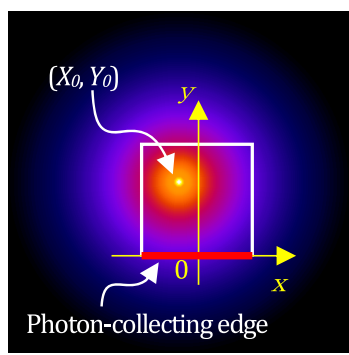

(c)

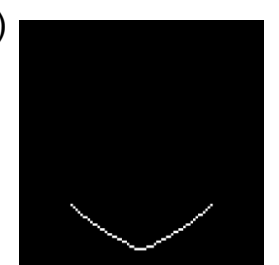

Fig. 7 Procedure for calculating an ECF. (a) The radial distribution is extended to one in the Cartesian coordinate system. It is shifted under a perimeter of the LWG (a square in this example). The efficiency is integrated along the whole perimeter and along a specified section of its perimeter. For example, two curves to be used for a heart-shaped LWG are shown in (b) and (c). 


\subsection{Numerical Example}

The first example is a square LWG. Its thickness is set to either 10 or $4 \mathrm{~mm}$ while the transmittance at $450 \mathrm{~nm}$ is fixed at 0.1 . Either the bottom edge or the whole perimeter is specified as the photon-collecting edge. The perimeter of an LWG is specified by drawing a hollow square with a software such as Microsoft Paint. This image $(100 \times 100$ pixels $)$ is converted to a text image file by ImageJ (a general-purpose image processing program developed by the National Institutes of Health). The photon-collecting edge is set up by repeating this process. Our in-house Fortran90 program reads these text files and other data files for the materials and geometrical parameters and calculates the ECF. The result is shown in Fig. 8. Each color-coded image represents an ECF for an LWG with its thickness and the collecting edge as indicated. To show subtle changes in the efficiency distribution, only a limited range of the efficiency values is shown, as indicated below each image. Because these values are probabilities, they are dimensionless.

When the photons are collected by the bottom edge only, the efficiency decreases as the excited spot moves away from the bottom edge. This is partly because the escape-cone is not fully developed in the region near the bottom edge and partly because the PL photons spread in the three-dimensional space. Comparison of Figs. 8(a) and 8(c) shows that the efficiency of the 4-mm-thick LWG decreases more rapidly. When the whole perimeter is specified as the collecting edge, the ECF is invariant under point reflection through its center as shown in Figs. 8(b) and 8(d). These ECFs are much more uniform. The 4-mm-thick LWG has lower efficiency due to the self-absorption loss.

We repeat this procedure for hexagonal and heart-shaped LWGs. The result is summarized in Figs. 9 and 10, respectively. The efficiency outside the LWGs is set equal to the minimum value in this color-coded presentation. The same trends are observed in these ECFs as in Fig. 8. The efficiency decreases as the excited spot moves away from the collecting edge. It decreases more rapidly in the thinner LWGs due to enhanced self-absorption. When the whole perimeter is specified as the collecting edge, ECFs possess central symmetry and become more uniform. Subtle variations are observed in Figs. 9(b), 9(d), 10(b), and 10(d) because only a small range of efficiency values is shown. This difference is caused by the interplay between multiple effects: geometrical spreading of light, self-absorption, and imperfect formation of escape-cones near the

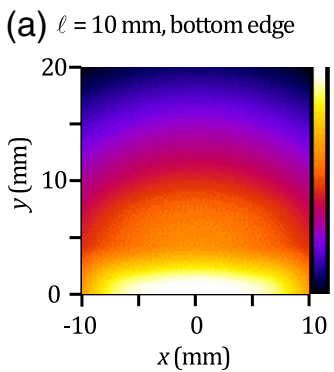

Color scale: 0.152 to 0.199

(c) $\ell=4 \mathrm{~mm}$, bottom edge

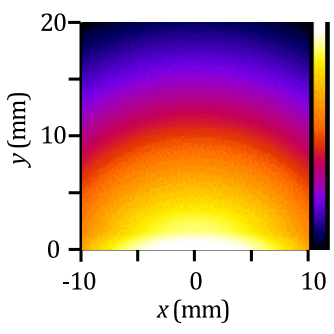

Color scale: 0.113 to 0.175 (b) $\ell=10 \mathrm{~mm}$, all edges

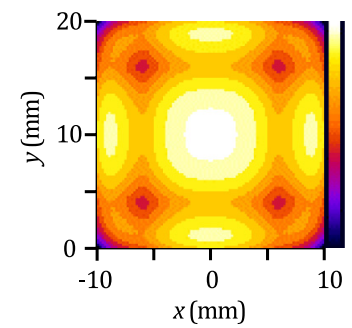

Color scale: 0.685 to 0.686

(d) $\ell=4 \mathrm{~mm}$, all edges

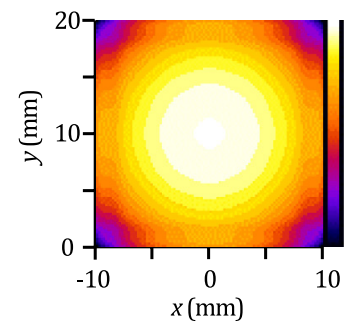

Color scale: 0.528 to 0.567

Fig. 8 ECFs for square LWGs. In (a) and (c), the bottom edge is the photon-collecting edge. In (b) and (d), photons are collected by all four edges. The thickness $\ell$ is as indicated. 
Fujieda and Tsutsumi: Extending the concept of edge collection function to polygonal...

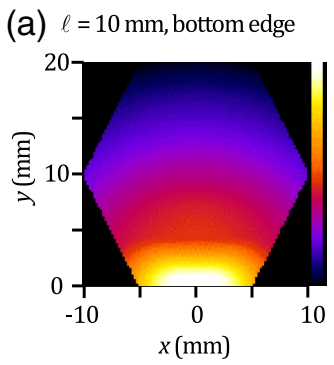

Color scale: 0.150 to 0.210

(c) $\ell=4 \mathrm{~mm}$, bottom edge

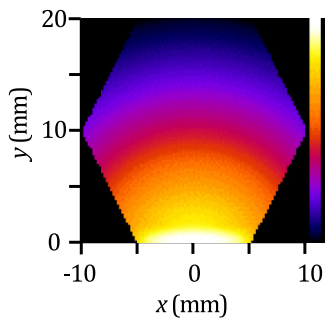

Color scale: 0.114 to 0.186 (b) $\ell=10 \mathrm{~mm}$, all edges

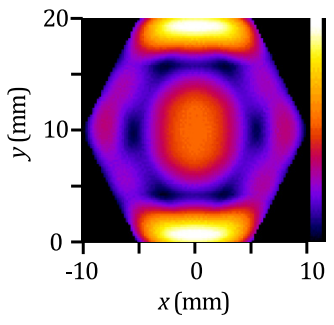

Color scale: 0.688 to 0.707

(d) $\ell=4 \mathrm{~mm}$, all edges

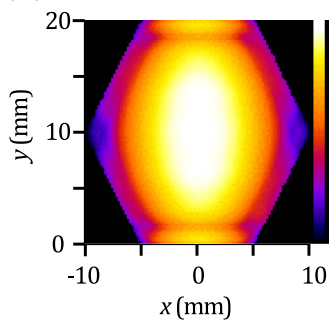

Color scale: 0.562 to 0.588

Fig. 9 ECFs for hexagonal LWGs. The thickness $\ell$ is as indicated. In (a) and (c), only the bottom edge is specified as the photon-collecting edge. In (b) and (d), photons are collected by the whole perimeter.

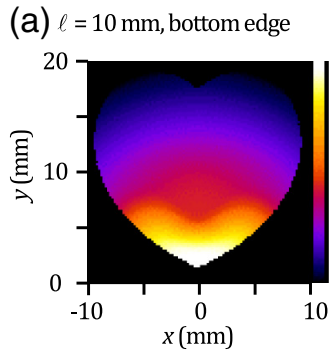

Color scale: 0.1142 to 0.186

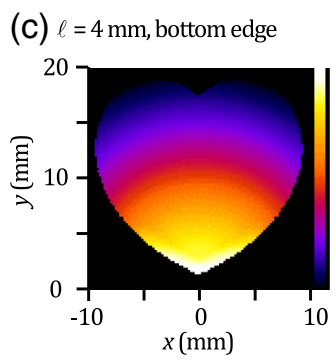

Color scale: 0.110 to 0.164 (b) $\ell=10 \mathrm{~mm}$, all edges

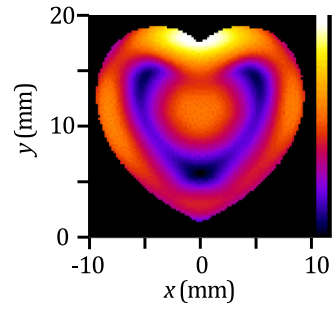

Color scale: 0.694 to 0.716

(d) $\ell=4 \mathrm{~mm}$, all edges

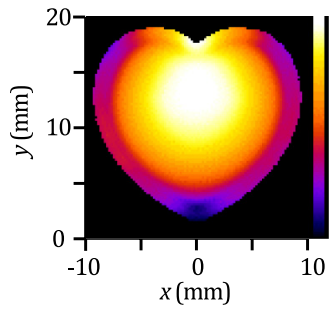

Color scale: 0.573 to 0.603

Fig. 10 ECFs for heart-shaped LWGs. The thickness $\ell$ is as indicated. For the ECFs in (a) and (c), the photon-collecting edge is set to the bottom section of the perimeter as shown in Fig. 7(c). In (b) and (d), photons are collected by the whole perimeter.

perimeter. The reason that the ECFs in Figs. 9(b) and 9(d) are invariant only for a $180^{\circ}$ rotation is that our drawing of the hexagon is slightly elongated.

\section{Conclusions}

LWGs have been studied for LSCs, BIPV systems, energy-harvesting displays, and large-area sensors. In general, the light incident on an LWG is not uniform except for the case of LSCs 
Fujieda and Tsutsumi: Extending the concept of edge collection function to polygonal...

installed in a place with no light-blocking structures. An advanced model should be able to handle non-uniform irradiation on LWGs with various shapes. We formulated optical efficiency of polygonal and curved planar LWGs as follows. Monochromatic light excites a single spot in an LWG, and PL photons are generated. We assume isotropic emission from the material although emission from the luminescent layer is anisotropic due to self-absorption. Scattering and re-emission events are neglected for simplicity. First, optical efficiency is expressed as a function of the radius in cylindrical geometry. Three photon-loss mechanisms are considered: leakage from the top and bottom surfaces without reflection at the air-LWG interface, leakage after being trapped in the LWG, and self-absorption during the wave-guiding process. Second, this efficiency curve is converted to a distribution in the Cartesian coordinate system. Optical efficiency is calculated from the line integral of this distribution along a specified, photon-collecting edge of an LWG. By translating the efficiency distribution and repeating this calculation, an ECF is known for all points on the LWG. Now, one can calculate the response of an LWG to an arbitrary incident radiation pattern. For example, we calculated ECFs of square, hexagonal, and heart-shaped LWGs and showed how they depend on the LWG thickness.

This analysis might be useful for predicting the performance of polygonal and curved LWGs under non-uniform illumination. Because semi-transparent LWGs with various colors and shapes have aesthetic values, they might also be suited for powering wearable electronic devices.

\section{Appendix: Relative Strength of Re-Emission to Initial Photoluminescence}

In many experiments on LSCs, re-emission and initial PL events are indistinguishable. In the single-spot excitation experiment described in Ref. 28, re-emission events were directly observed because they were spatially separated from the initial emission. The camera was placed $20 \mathrm{~cm}$ away from the 5-cm-square LWG. This ensured that the solid angle subtended by the camera was approximately the same for every emission spot on the LWG. Therefore, the observed intensity distribution is proportional to the number of the PL photons propagating in the LWG.

Analysis on the images acquired by the camera allows us to roughly estimate the relative strength of these emissions. This is accomplished as follows. First, a circular region of interest (ROI) is set around the excited spot in the image acquired with a short exposure time $(0.025 \mathrm{~ms})$ as shown in the enlarged image in Fig. 11(a). The total pixel value in this ROI divided by the exposure time is proportional to the number of the initial PL photons. Next, the ROI radius is increased to encompass the concentric re-emission pattern in the image acquired with a long exposure time $(1000 \mathrm{~ms})$ as shown in Fig. 11(b). The re-emission intensity distribution peaks at the radius equal to $\ell \tan \theta_{c}$, where $\ell$ is the LWG thickness and $\theta_{c}$ is the critical angle for TIR. ${ }^{28}$ Because the intensity diminishes at a larger radius, the total pixel value in the circular ROI reaches an asymptotic value. This value divided by the exposure time is proportional to the number of re-emitted photons in addition to that of the initial PL photons. The latter contribution is subtracted by adding the pixel values in the ROI with a radius slightly smaller than $\ell \tan \theta_{c}$, which is fixed to $7.47 \mathrm{~mm}$ in Fig. 11(b).

This analysis is repeated by varying the ROI radius for the two images recorded with a 50-mm-square and 10-mm-thick LWG. The result is summarized as a function of the ROI radius in Fig. 12. The ordinate indicates the total pixel value divided by the exposure time. This index for the initial emission (blue circle) slightly increases with the ROI radius due to the small reemission contribution. Namely, about $4 \%$ of the initial PL photons with near-vertical emission angles are reflected back and can generate re-emission in the region near the excited spot. The index for the re-emission events (red triangle) gradually increases with the ROI radius and approaches a plateau. From the ratio of these two indices, we conclude that the total number of re-emitted PL photons is about 2 orders of magnitude smaller than that of the PL photons emitted initially. The luminescent material used in this experiment is Lumogen F Red 305. Its quantum efficiency is reported to be close to unity. ${ }^{30}$ It is reasonable to expect that re-emission contributes less for a material with a smaller quantum efficiency. 
Fujieda and Tsutsumi: Extending the concept of edge collection function to polygonal...

(a)

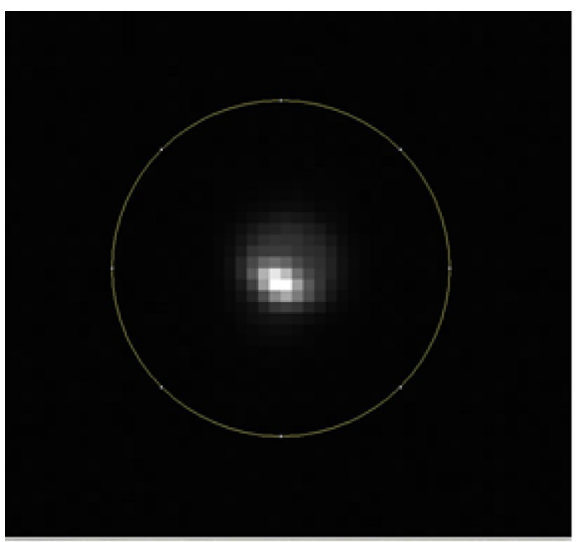

(b)
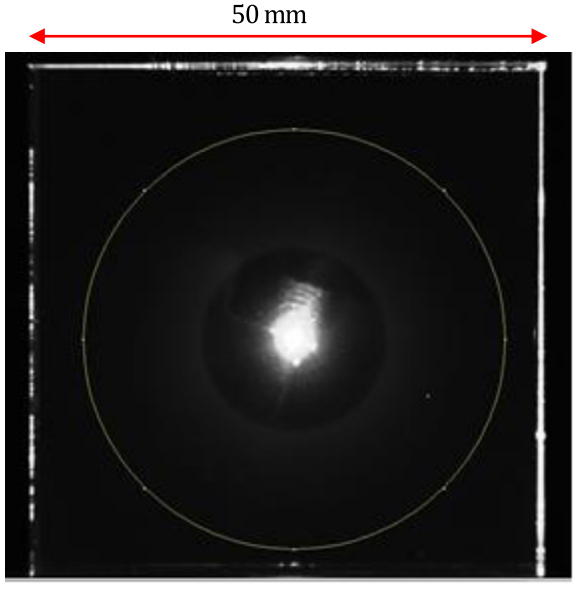

Fig. 11 Analysis on the images acquired by the camera in the single-spot excitation experiment. (a) An enlarged image acquired with 0.025 -ms exposure time shows the intensity distribution of the initial emission. A ring of radius $=0.77 \mathrm{~mm}$ is added for estimating the magnitude of the initial emission. (b) The image acquired with 1000-ms exposure time. The radius is increased to $20.6 \mathrm{~mm}$ to encompass most of the re-emission region.

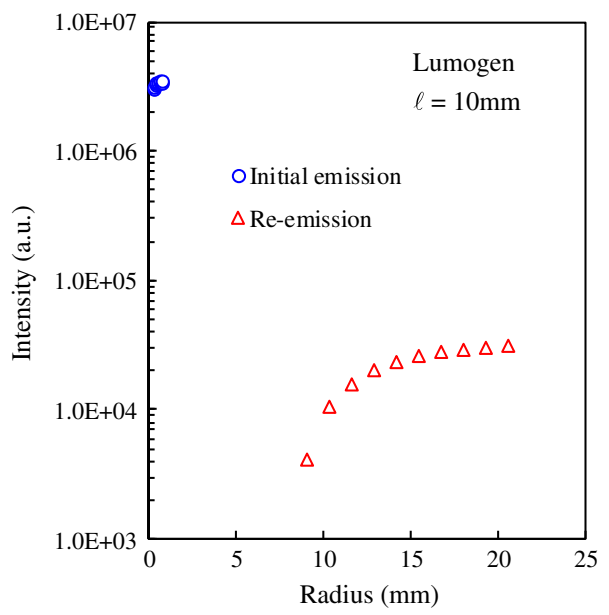

Fig. 12 Relative magnitude of the initial emission and re-emission extracted from the two images acquired with different exposure times. 


\section{Acknowledgments}

This work was supported by the Japan Society for the Promotion of Science; Award No. $17 \mathrm{~K} 07032$.

\section{References}

1. W. H. Weber and J. Lambe, "Luminescent greenhouse collector for solar radiation," Appl. Opt. 15(10), 2299-2300 (1976).

2. J. S. Batchelder, A. H. Zewai, and T. Cole, "Luminescent solar concentrators. 1: theory of operation and techniques for performance evaluation," Appl. Opt. 18(18), 3090-3110 (1979).

3. R. W. Olson, R. F. Loring, and M. D. Fayer, "Luminescent solar concentrators and the reabsorption problem," Appl. Opt. 20(17), 2934-2940 (1981).

4. M. V. Mukhina et al., "Electrically controlled polarized photoluminescence of CdSe/ZnS nanorods embedded in a liquid crystal template," Nanotechnology 23, 325201 (2012).

5. N. D. Bronstein et al., "Luminescent solar concentration with semiconductor nanorods and transfer-printed micro-silicon solar cells," ACS Nano 8(1) 44-53 (2014).

6. F. Meinardi et al., "Highly efficient large-area colourless luminescent solar concentrators using heavy-metal-free colloidal quantum dots," Nat. Nanotechnol. 10, 878-885 (2015).

7. L. H. Slooff et al., "A luminescent solar concentrator with $7.1 \%$ power conversion efficiency," Phys. Stat. Sol. (RRL) 2(6), 257-259 (2008).

8. F. Meinardi, F. Bruni, and S. Brovelli, "Luminescent solar concentrators for buildingintegrated photovoltaics," Nat. Rev. Mater. 2, 17072 (2017).

9. C. L. Mulder et al., "Dye alignment in luminescent solar concentrators: II. Horizontal alignment for energy harvesting in linear polarizers," Opt. Express 18, A91-A99 (2010).

10. I. Fujieda et al., "Energy-harvesting laser phosphor display and its design considerations," J. Photonics Energy 7(2), 028001 (2017).

11. K. Yunoki et al., "Crosstalk and optical efficiency of an energy-harvesting color projector utilizing ceramic phosphors," Appl. Opt. 58(36), 9896-9903 (2019).

12. P. P. Manousiadis et al., "Wide field-of-view fluorescent antenna for visible light communications beyond the étendue limit," Optica 3, 702-706 (2016).

13. D. Guberman et al., "The light-trap: a novel concept for a large SiPM-based pixel for very high energy gamma-ray astronomy and beyond," Nucl. Instrum. Methods Phys. Res. A 923, 19-25 (2019).

14. Y. Tsutsumi et al., "Position-sensitive detectors based on redshifts in photoluminescence spectra," Opt. Eng. 58(7), 077108 (2019).

15. J. Sansregret et al., "Light transport in planar luminescent solar concentrators: the role of DCM self-absorption," Appl. Opt. 22(4), 573-577 (1983).

16. I. Sychugov, "Analytical description of a luminescent solar concentrator," Optica 6, 1046-1049 (2019).

17. I. Fujieda et al., "Edge collection function: an analytical expression for the optical efficiency of an energy-harvesting device based on photoluminescence," Opt. Eng. 58(10), 104101 (2019).

18. E. Loh and D. J. Scalapino, "Luminescent solar concentrators: effects of shape on efficiency," Appl. Opt. 25, 1901-1907 (1986).

19. A. Goetzberger, "Fluorescent solar energy collectors: operating conditions with diffuse light," Appl. Phys. 16, 399-404 (1978).

20. M. S. De Cardona et al., "Edge effect on luminescent solar concentrators," Solar Cells 15, 225-230 (1985).

21. R. Soti et al., "Photon transport in luminescent solar concentrators," J. Lumin. 68, 105-114 (1996).

22. O. V. Sulima et al., "AlGaAs photovoltaic arrays spectrally matched to photoluminescent fibers for UGS application," Proc. SPIE 5090, 360-368 (2003).

23. O. Y. Edelenbosch et al., "Luminescent solar concentrators with fiber geometry," Opt. Express 21, A503-A514 (2013). 
24. K. R. McIntosh, N. Yamada, and B. S. Richards, "Theoretical comparison of cylindrical and square-planar luminescent solar concentrators," Appl. Phys. B 88, 285-290 (2007).

25. J. J. H. Videira, E. Bilotti, and A. J. Chatten, "Cylindrical array luminescent solar concentrators: performance boosts by geometric effects," Opt. Express 24, A1188-A1200 (2016).

26. A. Reinders, M. G. Debije, and A. Rosemann, "Measured efficiency of a luminescent solar concentrator PV module called leaf roof," IEEE J. Photovoltaics 7(6), 1663-1666 (2017).

27. R. Matsumura, Y. Tsutsumi, and I. Fujieda, "Concentric re-emission pattern from a planar waveguide with a thin uniform luminescent layer," Appl. Opt. 59(6), 1703-1710 (2020).

28. I. Fujieda and M. Ohta, "Angle-resolved photoluminescence spectrum of a uniform phosphor layer," AIP Adv. 7, 105223 (2017).

29. O. M. ten Kate, K. M. Hooning, and E. van der Kolk, "Quantifying self-absorption losses in luminescent solar concentrators," Appl. Opt. 53(23), 5238-5245 (2014).

30. L. R. Wilson and B. S. Richards, "Measurement method for photoluminescent quantum yields of fluorescent organic dyes in polymethyl methacrylate for luminescent solar concentrators," Appl. Opt. 48(2), 212-220 (2009).

Ichiro Fujieda is a professor at Ritsumeikan University. He received his $\mathrm{PhD}$ from the University of California, Berkeley, in 1990. He was with Xerox Palo Alto Research Center from 1990 to 1992 and NEC Corporation from 1992 to 2003.

Yasuhiro Tsutsumi is an assistant professor at Ritsumeikan University. He received BE and ME degrees and his PhD from Osaka Prefecture University in 2007, 2009, and 2012, respectively. His current research interests include optical sensing, optical fiber devices, and optical communications. 